\title{
NUMERICAL ANALYSIS OF THE MIXED BOUNDARY VALUE PROBLEM FOR THE SOPHIE GERMAIN EQUATION
}

\author{
A. L. Ushakov, South Ural State University, Chelyabinsk, Russian Federation, \\ ushakoval@susu.ru.
}

The Sophie Germain equation in variational form is considered on a plane bounded domain under mixed boundary conditions. The original problem is continued to a rectangular domain along the boundary with the Dirichlet boundary conditions. The continued problem is discretized using the finite element method. We present a method of fictitious components, which, for the absolute error, has a convergence rate as a geometric progression. For the numerical solution of the continued problem, we use the method of iterative extensions, which is obtained as a generalization of the method of fictitious components. For the relative error, the method of iterative extensions has the same rate of convergence as a geometric progression in a stronger norm than the energy norm of the problem. The choice of iterative parameters is based on the minimum residual method. An algorithmic implementation of the iterative extension method with automatic selection of iterative parameters is presented. The algorithm also provides a criterion for automatically stopping the iterative process when a given estimate of the relative error is reached. The software implementation of this algorithm does not require additional complications associated with the complexity of the geometry of the domain, the order of the differential equation and the presence of Dirichlet boundary conditions. We consider an example of calculations by the method of iterative extensions within the numerical solution of a particular problem. In order to perform computations, we use the condition of evaluating the relative error in a norm, which is stronger than the norm generated by the problem operator. But the relative errors for the obtained numerical solution to the example of the original problem are considered in other ways. For example, a point-by-point calculation of the relative error is carried out for the grid points. It turns out that several iterations are enough to have a relative error of only a few percent, even with different methods of calculating the error. In practice, computational experiments confirm the optimality of the method indicated in theory.

Keywords: Sophie Germain equation; method of fictitious components; method of iterative extensions.

\section{Introduction}

Let us consider the Sophie Germain equation in variational form with Dirichlet mixed boundary conditions, symmetry and hinge conditions, Neumann conditions in a bounded domain on the plane. We present this original problem in the first domain, and consider its fictitious continuation in the second domain in order to obtain a problem extended across the boundary with Dirichlet conditions in a rectangular domain. In the presented approach, we try to eliminate the additional complication of the main problems arising in the numerical solution of elliptic boundary value problems in bounded domains associated with the geometry of the domain, the order of the differential equation and the presence of Dirichlet boundary conditions [1-5]. We consider numerical methods that are both resistant to computational errors when computing on a computer and asymptotically optimal in terms of computational complexity in arithmetic operations, as well as quite universal, 
and have a simple software implementation of the computational algorithm. To this end, consider a generalization of the method of fictitious components in the form of the method of iterative extensions, which does not significantly complicate the process of numerical solution of the original problem in comparison with the known approaches [4-7]. Note that we reduce the solution of the original problem under consideration to the solution of problems, in the numerical solution of which we can apply, for example, well-known marching methods that are optimal in terms of computational complexity [8-11].

\section{Boundary Value Problem in Variational Form}

Let us complete the domain for solving the original problem to a rectangular domain in the first domain on the plane with the help of the second domain.

$$
\bar{\Omega}_{1} \cup \bar{\Omega}_{\mathrm{II}}=\bar{\Pi}, \Omega_{1} \cap \Omega_{\mathrm{II}}=\emptyset, \Omega_{\omega} \subset \mathrm{R}^{2}, \omega \in\{1, \mathrm{II}\} .
$$

The boundaries of these domains consist of the closure of unions of open nonintersecting parts.

$$
\begin{gathered}
\partial \Pi=\bar{s}, s=\Gamma_{0} \cup \Gamma_{1} \cup \Gamma_{2} \cup \Gamma_{3}, \Gamma_{i} \cap \Gamma_{j}=\emptyset, i \neq j, i, j=0,1,2,3, \\
\partial \Omega_{\omega}=\bar{s}_{\omega}, s_{\omega}=\Gamma_{\omega, 0} \cup \Gamma_{\omega, 1} \cup \Gamma_{\omega, 2} \cup \Gamma_{\omega, 3}, \Gamma_{\omega, i} \cap \Gamma_{\omega, j}=\emptyset, i \neq j, i, j=0,1,2,3 .
\end{gathered}
$$

We assume that a non-empty intersection of the boundary of the first domain and the boundary of the second domain is the closure of the intersection of the corresponding parts of the boundaries of these domains.

$$
\partial \Omega_{1} \cap \partial \Omega_{\mathrm{II}}=\bar{S}, S=\Gamma_{1,0} \cap \Gamma_{\mathrm{II}, 3} \neq \emptyset .
$$

We consider the original mixed boundary value problem for the Sophie Germain equation in the first domain. Additionally, in the second domain, we introduce a fictitious mixed boundary value problem for the homogeneous screened Sophie Germain equation.

$$
\breve{u}_{\omega} \in \breve{H}_{\omega}: \Lambda_{\omega}\left(\breve{u}_{\omega}, \breve{v}_{\omega}\right)=F_{\omega}\left(\breve{v}_{\omega}\right) \forall \breve{v}_{\omega} \in \breve{H}_{\omega} .
$$

The spaces of solutions to such problems are the following Sobolev spaces.

$$
\breve{H}_{\omega}=\breve{H}_{\omega}\left(\Omega_{\omega}\right)=\left\{\breve{v}_{\omega} \in W_{2}^{2}\left(\Omega_{\omega}\right):\left.\breve{v}\right|_{\Gamma_{\omega, 0} \cup \Gamma_{\omega, 1}}=0,\left.\frac{\partial \breve{v}_{\omega}}{\partial n}\right|_{\Gamma_{\omega, 0} \cup \Gamma_{\omega, 2}}=0\right\} .
$$

The right-hand sides of the problems are linear functionals.

$$
F_{\omega}\left(\breve{v}_{\omega}\right)=\left(\breve{f}_{\omega}, \breve{v}_{\omega}\right),\left(\breve{f}_{\omega}, \breve{v}_{\omega}\right)=\int_{\Omega_{\omega}} \breve{f}_{\omega} \breve{v}_{\omega} d \Omega_{\omega}, \breve{f}_{\text {II }}=0 .
$$

The left-hand sides of the problems are bilinear forms.

$$
\begin{gathered}
\Lambda_{\omega}\left(\breve{u}_{\omega}, \breve{v}_{\omega}\right)=\int_{\Omega_{\omega}}\left(\sigma_{\omega} \Delta \breve{u}_{\omega} \Delta \breve{v}_{\omega}+\left(1-\sigma_{\omega}\right)\left(\breve{u}_{\omega x x} \breve{v}_{\omega y e}+2 \breve{u}_{\omega x y} \breve{v}_{\omega x y}+\breve{u}_{\omega y y} \breve{v}_{\omega y y}\right)+a_{\omega} \breve{u}_{\omega} \breve{v}_{\omega}\right) d \Omega_{\omega}, \\
\sigma_{\omega} \in(0 ; 1), a_{1}=0, a_{\mathrm{II}} \geq 0 .
\end{gathered}
$$

We assume that, in the considered spaces, bilinear forms give normalizations equivalent to those of the Sobolev spaces used.

$$
\exists c_{1}, c_{2}>0: c_{1}\left\|\breve{v}_{\omega}\right\|_{W_{2}^{2}\left(\Omega_{\omega}\right)}^{2} \leq \Lambda_{\omega}\left(\breve{\nu}_{\omega}, \breve{v}_{\omega}\right) \leq c_{2}\left\|\breve{v}_{\omega}\right\|_{W_{2}^{2}\left(\Omega_{\omega}\right)}^{2} \forall \breve{v}_{\omega} \in \breve{H}_{\omega}
$$

These assumptions imply the existence, uniqueness of the solution to the original problem and only zero solution to the fictitious problem. 


\section{Continued Problem}

Simultaneous consideration of the original and fictitious problems is possible. We refer to this problem as a continuation problem.

$$
\breve{u} \in \breve{V}: \quad \Lambda_{1}\left(\breve{u}, I_{1} \breve{v}\right)+\Lambda_{\mathrm{II}}(\breve{u}, \breve{v})=F_{1}\left(I_{1} \breve{v}\right) \forall \breve{v} \in \breve{V} .
$$

For this problem, the extended solution space is the Sobolev space.

$$
\breve{V}=\breve{V}(\Pi)=\left\{\breve{v} \in W_{2}^{2}(\Pi):\left.\breve{v}\right|_{\Gamma_{0} \cup \Gamma_{1}}=0,\left.\frac{\partial \breve{v}}{\partial n}\right|_{\Gamma_{0} \cup \Gamma_{2}}=0\right\} .
$$

The extended solution space contains the solution subspace of the continued problem, the solution space of the original problem on the first domain, which is continued by zero in order to complete the first domain to the rectangular domain.

$$
\breve{V}_{1}=\breve{V}_{1}(\Pi)=\left\{\breve{v}_{1} \in \breve{V}:\left.\breve{v}_{1}\right|_{\Pi \backslash \Omega_{1}}=0\right\} .
$$

In the continued problem, we use the operator, which is, generally speaking, of a nonorthogonal projection of the extended space onto the subspace of solutions to the continued problem.

$$
I_{1}: \breve{V} \mapsto \breve{V}_{1}, \breve{V}_{1}=i m I_{1}, I_{1}=I_{1}^{2} .
$$

Let us introduce the subspaces of the extended solution space.

$$
\begin{gathered}
\breve{V}_{3}=\breve{V}_{3}(\Pi)=\left\{\breve{v}_{3} \in \breve{V}:\left.\breve{v}_{3}\right|_{\Pi \backslash \Omega_{\mathrm{II}}}=0\right\}, \\
\breve{V}_{2}=\breve{V}_{2}(\Pi)=\left\{\breve{v}_{2} \in \breve{V}: \Lambda\left(\breve{v}_{1}, \breve{v}_{2}\right)=0 \forall \breve{v}_{1} \in \breve{V}_{1}, \Lambda\left(\breve{v}_{2}, \breve{v}_{3}\right)=0 \forall \breve{v}_{3} \in \breve{V}_{3}\right\}, \\
\breve{V}=\breve{V}_{1} \oplus \breve{V}_{\mathrm{II}}, \breve{V}_{\mathrm{II}}=\breve{V}_{2} \oplus \breve{V}_{3} .
\end{gathered}
$$

Here the new bilinear form is the sum of the previous bilinear forms.

$$
\Lambda(\breve{u}, \breve{v})=\Lambda_{1}(\breve{u}, \breve{v})+\Lambda_{\mathrm{II}}(\breve{u}, \breve{v}) \forall \breve{u}, \breve{v} \in \breve{V}
$$

We assume that the bilinear form gives a normalization of the extended solution space equivalent to the normalization of the Sobolev space.

$$
\exists c_{1}, c_{2}>0: c_{1}\|\breve{v}\|_{W_{2}^{2}(\Pi)}^{2} \leq \Lambda(\breve{v}, \breve{v}) \leq c_{2}\|\breve{v}\|_{W_{2}^{2}(\Pi)}^{2} \forall \breve{v} \in \breve{V} .
$$

We use the Sobolev spaces, in which there is fulfillment of the assumptions about the continuation of functions in the form

$$
\exists \breve{\beta}_{1} \in(0 ; 1], \breve{\beta}_{2} \in\left[\breve{\beta}_{1} ; 1\right]: \breve{\beta}_{1} \Lambda\left(\breve{v}_{2}, \breve{v}_{2}\right) \leq \Lambda_{\mathrm{II}}\left(\breve{v}_{2}, \breve{v}_{2}\right) \leq \breve{\beta}_{2} \Lambda\left(\breve{v}_{2}, \breve{v}_{2}\right) \forall \breve{v}_{2} \in \breve{V}_{2}
$$

Then, the solution to the continued problem exists and is unique. This is the solution to the original problem on the first domain with zero continuation on the rest part of the rectangular domain. Note that the solution to the original problem and the solution to the original problem continued by zero, i.e. the solution to the continued problem, can be denoted in the same way as a function and its continuation.

$$
\breve{H}_{\omega}\left(\Omega_{\omega}\right)=\breve{V}_{\omega}\left(\Omega_{\omega}\right) .
$$




\section{Discretization of the Continued Problem}

Consider the discretization of the continued problem with a specific type of boundary conditions.

$$
\begin{gathered}
\Pi=\left(0 ; b_{1}\right) \times\left(0 ; b_{2}\right), \Gamma_{0}=\emptyset, \Gamma_{1}=\left\{b_{1}\right\} \times\left(0 ; b_{2}\right) \cup\left(0 ; b_{1}\right) \times\left\{b_{2}\right\}, \\
\Gamma_{2}=\{0\} \times\left(0 ; b_{2}\right) \cup\left(0 ; b_{1}\right) \times\{0\}, \Gamma_{3}=\emptyset, b_{1}, b_{2} \in(0 ;+\infty) .
\end{gathered}
$$

Define a grid in the rectangular domain.

$$
\begin{gathered}
\left(x_{i} ; y_{j}\right)=\left((i-0,5) h_{1} ;(j-0,5) h_{2}\right), h_{1}=b_{1} /(m+0,5), h_{2}=b_{2} /(n+0,5), \\
i=1,2 \ldots, m, j=1,2 \ldots, n, m, n \in \mathrm{N} .
\end{gathered}
$$

Let us introduce grid functions on the set of nodes of the introduced grid.

$$
v_{i, j}=v\left(x_{i} ; y_{j}\right) \in \mathrm{R}, i=1,2 \ldots, m, j=1,2 \ldots, n, m, n \in \mathrm{N} .
$$

Let us apply the completion of grid functions using parabolic basis functions.

$$
\begin{gathered}
\Phi^{i, j}(x ; y)=\Psi^{1, i}(x) \Psi^{2, j}(y), i=1,2 \ldots, m, j=1,2 \ldots, n, m, n \in \mathrm{N}, \\
\Psi^{1, i}(x)=[1 / i] \Psi\left(x / h_{1}-i+3\right)+\Psi\left(x / h_{1}-i+3\right)+[i / m] \Psi\left(x / h_{1}-i\right), \\
\Psi^{2, j}(y)=[1 / j] \Psi\left(y / h_{2}-j+3\right)+\Psi\left(y / h_{2}-j+3\right)+[j / n] \Psi\left(y / h_{2}-j\right), \\
\Psi(z)=\left\{\begin{array}{cc}
0,5 z^{2}, & z \in[0 ; 1], \\
-z^{2}+3 z-1,5, & z \in[1 ; 2], \\
0,5 z^{2}-3 z-4,5, & z \in[2 ; 3], \\
0, & z \notin(0 ; 3) .
\end{array}\right.
\end{gathered}
$$

And we assume that the values of the basis functions are equal to zero outside the rectangular domain.

$$
\Phi^{i, j}(x ; y)=0, \quad(x ; y) \notin \Pi, i=1,2 \ldots, m, j=1,2 \ldots, n, m, n \in \mathrm{N} .
$$

Combinations of basic functions form a finite-dimensional subspace in the space of solutions to the extended problem.

$$
\tilde{V}=\left\{\sum_{i=1}^{m} \sum_{j=1}^{n} v_{i, j} \Phi^{i, j}(x ; y)\right\} \subset \breve{V} .
$$

We approximate the continued problem and obtain the linear system of equations

$$
\bar{u} \in \mathrm{R}^{N}: B \bar{u}=\bar{f}, \bar{f} \in \mathrm{R}^{N} .
$$

We assume that the projection operator nullifies the coefficients of the basis functions when their supports do not belong entirely to the first domain. We get the continued problem in the matrix form. Define the matrix of the discrete continued problem continued on the right in the system.

$$
\langle B \bar{u}, \bar{v}\rangle=\Lambda_{1}\left(\tilde{u}, I_{1} \tilde{v}\right)+\Lambda_{\mathrm{II}}(\tilde{u}, \tilde{v}) \forall \tilde{u}, \tilde{v} \in \tilde{V},\langle\bar{f}, \bar{v}\rangle=F_{1}\left(I_{1} \tilde{v}\right) \forall \tilde{v} \in \tilde{V},
$$




$$
\langle\bar{f}, \bar{v}\rangle=(\bar{f}, \bar{v}) h_{1} h_{2}=\bar{f} \bar{v} h_{1} h_{2}, \bar{v}=\left(v_{1}, v_{2}, \ldots, v_{N}\right)^{\prime} \in \mathrm{R}^{N}, N=m n .
$$

Here, first of all, we enumerate the basis functions with supports from the first domain. Second, we enumerate the basis functions with supports crossing the boundaries of both domains simultaneously. Third, we enumerate the basis functions with supports from the second domain. During this enumeration, the used vectors have the following structure

$$
\bar{v}=\left(\bar{v}_{1}^{\prime}, \bar{v}_{2}^{\prime}, \bar{v}_{3}^{\prime}\right)^{\prime}, \quad \bar{u}=\left(\bar{u}_{1}^{\prime}, \overline{0}^{\prime} \overline{0}^{\prime}\right)^{\prime}, \quad \bar{f}=\left(\bar{f}_{1}^{\prime}, \overline{0}^{\prime} \overline{0}^{\prime}\right)^{\prime} .
$$

Let us give formulas for calculating the elements of the matrix, the components of the vector from the right-hand side of the system, using the above order of enumeration of the basis functions.

$$
b_{i j}=h_{1}^{-1} h_{2}^{-1}\left(\Lambda_{1}\left(\Phi_{i}, I_{1} \Phi_{j}\right)+\Lambda_{\mathrm{II}}\left(\Phi_{i}, \Phi_{j}\right)\right), f_{i}=h_{1}^{-1} h_{2}^{-1} F_{1}\left(I_{1} \Phi_{i}\right), i, j=1,2, \ldots, N .
$$

\section{Method of Fictitious Components}

The well-known method of fictitious components can be written as follows.

$$
\begin{gathered}
\bar{u}^{k} \in \mathrm{R}^{N}: \Lambda\left(\bar{u}^{k}-\bar{u}^{k-1}\right)=-\tau_{k-1}\left(B \bar{u}^{k-1}-\bar{f}\right), k \in \mathrm{N}, \\
\forall \bar{u}^{0} \in \bar{V}_{1}, \tau_{0}=1 . \tau_{k-1}=2 /\left(\beta_{1}+\beta_{2}\right), k \in \mathrm{N} \backslash\{1\} .
\end{gathered}
$$

At any step of this iterative process, an extended problem, an extended matrix, arises.

$$
\langle\Lambda \bar{u}, \bar{v}\rangle=\Lambda(\tilde{u}, \tilde{v}) \forall \tilde{u}, \tilde{v} \in \tilde{V} .
$$

We calculate the elements of the matrix.

$$
l_{i j}=h_{1}^{-1} h_{2}^{-1} \Lambda\left(\Phi_{i}, \Phi_{j}\right), i, j=1,2, \ldots, N .
$$

The above matrices have the following structure.

$$
\Lambda=\left[\begin{array}{ccc}
\Lambda_{11} & \Lambda_{12} & 0 \\
\Lambda_{21} & \Lambda_{22} & \Lambda_{23} \\
0 & \Lambda_{32} & \Lambda_{33}
\end{array}\right], B=\left[\begin{array}{ccc}
\Lambda_{11} & \Lambda_{12} & 0 \\
0 & \Lambda_{02} & \Lambda_{23} \\
0 & \Lambda_{32} & \Lambda_{33}
\end{array}\right]
$$

Let us introduce the subspace of vectors.

$$
\bar{V}_{1}=\left\{\bar{v}=\left(\bar{v}_{1}^{\prime}, \bar{v}_{2}^{\prime}, \bar{v}_{3}^{\prime}\right)^{\prime} \in \mathrm{R}^{N}: \bar{v}_{2}=\overline{0}, \bar{v}_{3}=\overline{0}\right\} .
$$

Additionally, we define the vector subspaces.

$$
\begin{gathered}
\bar{V}_{3}=\left\{\bar{v}=\left(\bar{v}_{1}^{\prime}, \bar{v}_{2}^{\prime}, \bar{v}_{3}^{\prime}\right)^{\prime} \in \mathrm{R}^{N}: \bar{v}_{1}=\overline{0}, \bar{v}_{2}=\overline{0}\right\}, \\
\bar{V}_{2}=\left\{\bar{v}=\left(\bar{v}_{1}^{\prime}, \bar{v}_{2}^{\prime}, \bar{v}_{3}^{\prime}\right)^{\prime} \in \mathrm{R}^{N}: \Lambda_{11} \bar{v}_{1}+\Lambda_{12} \bar{v}_{2}=\overline{0}, \Lambda_{23} \bar{v}_{2}+\Lambda_{33} \bar{v}_{3}=\overline{0}\right\}, \mathrm{R}^{N}=\bar{V}_{1} \oplus \bar{V}_{2} \oplus \bar{V}_{3}, \\
\bar{V}_{\mathrm{I}}=\bar{V}_{1} \oplus \bar{V}_{2}, \bar{V}_{\mathrm{II}}=\bar{V}_{2} \oplus \bar{V}_{3} .
\end{gathered}
$$

We assume that when approximating the Sobolev space, the following inequalities hold for the continuation of functions.

$$
\exists \beta_{1} \in(0 ; 1], \beta_{2} \in\left[\beta_{1} ; 1\right]: \beta_{1}\left\langle\Lambda \bar{v}_{2}, \bar{v}_{2}\right\rangle \leq\left\langle B \bar{v}_{2}, \bar{v}_{2}\right\rangle \leq \beta_{2}\left\langle\Lambda \bar{v}_{2}, \bar{v}_{2}\right\rangle \forall \bar{v}_{2} \in \bar{V}_{2} .
$$


Let us define the norms generated by the extended matrix, its square and the identity matrix.

$$
\|\bar{v}\|_{\Lambda}=\sqrt{\langle\Lambda \bar{v}, \bar{v}\rangle},\|\bar{v}\|_{\Lambda^{2}}=\sqrt{\left\langle\Lambda^{2} \bar{v}, \bar{v}\right\rangle},\|\bar{v}\|=\sqrt{\langle\bar{v}, \bar{v}\rangle} \forall \bar{v} \in \mathrm{R}^{N} .
$$

Lemma 1. In method of fictitious components (4), the following estimate holds:

$$
\left\|\bar{u}^{1}-\bar{u}\right\|_{\Lambda^{2}} \leq 2\left\|\bar{u}^{0}-\bar{u}\right\|_{\Lambda^{2}} .
$$

Proof.

Let us denote the errors of the iterative process (4) by

$$
\bar{\psi}^{k}=\bar{u}^{k}-\bar{u}, k \in \mathrm{N} \cup\{0\} .
$$

We get equalities in the iterative process.

$$
\left(\Lambda\left(\bar{\psi}^{1}-\bar{\psi}^{0}\right)\right)^{2}=\left(-\Lambda_{11} \bar{\psi}_{1}^{0}\right)^{2}, \Lambda \bar{\psi}^{1} \Lambda \bar{\psi}^{1}-2 \Lambda \bar{\psi}^{1} \Lambda \bar{\psi}^{0}+\Lambda \bar{\psi}^{0} \Lambda \bar{\psi}^{0}=\Lambda_{11} \bar{\psi}_{1}^{0} \Lambda_{11} \bar{\psi}_{1}^{0} .
$$

Note the existence of the inequality

$$
\Lambda \bar{\psi}^{0} \Lambda \bar{\psi}^{0} \geq \Lambda_{11} \bar{\psi}_{1}^{0} \Lambda_{11} \bar{\psi}_{1}^{0} .
$$

We obtain inequalities

$$
\Lambda \bar{\psi}^{1} \Lambda \bar{\psi}^{1}-2 \Lambda \bar{\psi}^{1} \Lambda \bar{\psi}^{0} \leq 0,\left(\Lambda \bar{\psi}^{1} \Lambda \bar{\psi}^{1}\right)^{2} \leq\left(2 \Lambda \bar{\psi}^{1} \Lambda \bar{\psi}^{0}\right)^{2} \leq 4\left(\Lambda \bar{\psi}^{1} \Lambda \bar{\psi}^{1}\right)\left(\Lambda \bar{\psi}^{0} \Lambda \bar{\psi}^{0}\right) .
$$

Canceling, we obtain inequalities

$$
\Lambda \bar{\psi}^{1} \Lambda \bar{\psi}^{1} \leq 4 \Lambda \bar{\psi}^{0} \Lambda \bar{\psi}^{0},\left\|\bar{\psi}^{1}\right\|_{\Lambda^{2}} \leq 2\left\|\bar{\psi}^{0}\right\|_{\Lambda^{2}},\left\|\bar{u}^{1}-\bar{u}\right\|_{\Lambda^{2}} \leq 2\left\|\bar{u}^{0}-\bar{u}\right\|_{\Lambda^{2}} .
$$

Lemma 2. In iterative process (4), the following estimate with a positive value in the inequality holds:

$$
\left\|\bar{u}^{1}-\bar{u}\right\|_{\Lambda} \leq d\left\|\bar{u}^{1}-\bar{u}\right\|_{\Lambda^{2}} .
$$

The positive value in the inequality is estimated as an asymptotic equality

$$
d \approx\left(\lambda_{1,1}^{2}+a_{\mathrm{II}}\right)^{1 / 2} \lambda_{1,1}^{-2}, h_{1}, h_{2} \rightarrow 0, \lambda_{1,1}=\pi^{2}\left(b_{1}^{-2}+b_{2}^{-2}\right) / 4
$$

Proof.

Note that there exists the inequality with a positive value

$$
\exists d>0:\left(\Lambda \bar{\psi}^{1}, \bar{\psi}^{1}\right) \leq d^{2}\left(\Lambda \bar{\psi}^{1}, \Lambda \bar{\psi}^{1}\right) .
$$

Let us obtain an estimate for the indicated positive value in the inequality in the form of an asymptotic equality.

$$
\left(\left(\Delta^{2}+a_{\mathrm{II}}\right) \breve{\psi}^{1}, \breve{\psi}^{1}\right)=\sum_{i=1}^{+\infty} \sum_{j=1}^{+\infty}\left(\lambda_{i, j}^{2}+a_{\mathrm{II}}\right) c_{i, j}^{2}=\sum_{i=1}^{+\infty} \sum_{j=1}^{+\infty} \lambda_{i, j}^{2} c_{i, j}^{2}+\sum_{i=1}^{+\infty} \sum_{j=1}^{+\infty} a_{\mathrm{II}} c_{i, j}^{2} \leq
$$




$$
\begin{gathered}
\leq \frac{1}{\lambda_{1,1}^{2}} \sum_{i=1}^{+\infty} \sum_{j=1}^{+\infty} \lambda_{i, j}^{4} c_{i, j}^{2}+\frac{a_{\mathrm{II}}}{\lambda_{1,1}^{4}} \sum_{i=1}^{+\infty} \sum_{j=1}^{+\infty} \lambda_{i, j}^{4} c_{i, j}^{2}= \\
=\frac{\lambda_{1,1}^{2}+a_{\mathrm{II}}}{\lambda_{1,1}^{4}} \sum_{i=1}^{+\infty} \sum_{j=1}^{+\infty} \lambda_{i, j}^{4} c_{i, j}^{2}=\frac{\lambda_{1,1}^{2}+a_{\mathrm{II}}}{\lambda_{1,1}^{4}}\left(\Delta^{2} \breve{\psi}^{1}, \Delta^{2} \breve{\psi}^{1}\right) .
\end{gathered}
$$

Here we use solutions to the spectral problem.

$$
\breve{\psi}^{1}=\sum_{i=1}^{+\infty} \sum_{j=1}^{+\infty} c_{i, j} \breve{\varphi}_{i, j}, \quad\left(\breve{\varphi}_{i, j}, \breve{\varphi}_{i, j}\right)=1,\left(\breve{\varphi}_{i, j}, \breve{\varphi}_{p, l}\right)=0,(i ; j) \neq(p ; l), i, j, p, l \in \mathrm{N}
$$

where

$$
\begin{gathered}
\breve{\varphi}_{i, j} \in V\left(\left(0 ; b_{1}\right) \times\left(0 ; b_{2}\right)\right):-\Delta \breve{\varphi}_{i, j}=\lambda_{i, j} \breve{\varphi}_{i, j}, \breve{\varphi}_{i, j} \neq 0, \\
\lambda_{i, j}=0,25 \pi^{2}\left((2 i-1) b_{1}^{-2}+(2 j-1) b_{2}^{-2}\right), \quad i, j \in \mathrm{N} .
\end{gathered}
$$

Theorem 1. In method of fictitious components (4), convergence estimates

$$
\begin{gathered}
\left\|\bar{u}^{k}-\bar{u}\right\|_{\Lambda} \leq \varepsilon\left\|\bar{u}^{0}-\bar{u}\right\|_{\Lambda^{2}}=\varepsilon\left\|\bar{f}^{0}-\bar{f}\right\|, \\
\varepsilon=c q^{k-1}, c=2 d \in(0+\infty), k \in \mathrm{N}, \bar{f}^{0}=\mathrm{A} \bar{u}^{0}, 0 \leq q=\left(\beta_{2}-\beta_{1}\right) /\left(\beta_{1}+\beta_{2}\right)<1, \\
d \approx\left(\lambda_{1,1}^{2}+a_{\mathrm{II}}\right)^{1 / 2} \lambda_{1,1}^{-2}, h_{1}, h_{2} \rightarrow 0, \lambda_{1,1}=\pi^{2}\left(b_{1}^{-2}+b_{2}^{-2}\right) / 4
\end{gathered}
$$

hold. The absolute error of the method of fictitious components has a convergence rate in the energy norm not worse than the rate of convergence of a geometric progression.

\section{Method of Iterative Extensions}

To solve problem (3), we use a new method. Let us introduce the matrices

$$
\left\langle\Lambda_{\mathrm{I}} \bar{u}, \bar{v}\right\rangle=\Lambda_{1}(\tilde{u}, \tilde{v}),\left\langle\Lambda_{\mathrm{II}} \bar{u}, \bar{v}\right\rangle=\Lambda_{\mathrm{II}}(\tilde{u}, \tilde{v}),
$$

which have the following structure.

$$
\Lambda_{\mathrm{I}}=\left[\begin{array}{ccc}
\Lambda_{11} & \Lambda_{12} & 0 \\
\Lambda_{21} & \Lambda_{20} & 0 \\
0 & 0 & 0
\end{array}\right], \Lambda_{\mathrm{II}}=\left[\begin{array}{ccc}
0 & 0 & 0 \\
0 & \Lambda_{02} & \Lambda_{23} \\
0 & \Lambda_{32} & \Lambda_{33}
\end{array}\right]
$$

Define the extended matrix as the sum of the first matrix and the second matrix multiplied by a positive parameter.

$C=\mathrm{A}_{\mathrm{I}}+\gamma \mathrm{A}_{\mathrm{II}},\left[\begin{array}{ccc}C_{11} & C_{12} & 0 \\ C_{21} & C_{22} & C_{23} \\ 0 & C_{32} & C_{33}\end{array}\right]=\left[\begin{array}{ccc}\Lambda_{11} & \Lambda_{12} & 0 \\ \Lambda_{21} & \Lambda_{20} & 0 \\ 0 & 0 & 0\end{array}\right]+\gamma\left[\begin{array}{ccc}0 & 0 & 0 \\ 0 & \Lambda_{02} & \Lambda_{23} \\ 0 & \Lambda_{32} & \Lambda_{33}\end{array}\right], \gamma \in(0 ;+\infty)$

Assume that the statements on the continuation of functions, written in the following form, are fulfilled.

$\exists \beta_{1} \in(0 ;+\infty), \beta_{2} \in\left[\beta_{1} ;+\infty\right): \beta_{1}^{2}\left\langle C \bar{v}_{2}, C \bar{v}_{2}\right\rangle \leq\left\langle\Lambda_{\mathrm{II}} \bar{v}_{2}, \Lambda_{\mathrm{II}} \bar{v}_{2}\right\rangle \leq \beta_{2}^{2}\left\langle C \bar{v}_{2}, C \bar{v}_{2}\right\rangle \forall \bar{v}_{2} \in \bar{V}_{2}$, 


$$
\exists \alpha \in(0 ;+\infty):\left\langle\Lambda_{\mathrm{I}} \bar{v}_{2}, \Lambda_{\mathrm{I}} \bar{v}_{2}\right\rangle \leq \alpha^{2}\left\langle\Lambda_{\mathrm{II}} \bar{v}_{2}, \Lambda_{\mathrm{II}} \bar{v}_{2}\right\rangle \forall \bar{v}_{2} \in \bar{V}_{2}
$$

The iterative extension method is a generalization of the method of fictitious components, when an additional parameter is used when choosing an extended matrix, and the iterative parameters are selected based on the minimum residual method.

$$
\begin{gathered}
\bar{u}^{k} \in \mathrm{R}^{N}: C\left(\bar{u}^{k}-\bar{u}^{k-1}\right)=-\tau_{k-1}\left(B \bar{u}^{k-1}-\bar{f}\right), k \in \mathrm{N}, \\
\forall \bar{u}^{0} \in \bar{V}_{1}, \gamma>\alpha, \tau_{0}=1, \tau_{k-1}=\left\langle\bar{r}^{k-1}, \bar{\eta}^{k-1}\right\rangle /\left\langle\bar{\eta}^{k-1}, \bar{\eta}^{k-1}\right\rangle, k \in \mathrm{N} \backslash\{1\} .
\end{gathered}
$$

To calculate the iterative parameters, we sequentially calculate the residuals, corrections, and equivalent residuals.

$$
\bar{r}^{k-1}=B \bar{u}^{k-1}-\bar{f}, \bar{w}^{k-1}=C^{-1} \bar{r}^{k-1}, \bar{\eta}^{k-1}=B \bar{w}^{k-1}, k \in \mathrm{N} .
$$

Lemma 3. For iterative process (5), the following estimate holds:

$$
\left\|\bar{u}^{1}-\bar{u}\right\|_{C^{2}} \leq 2\left\|\bar{u}^{0}-\bar{u}\right\|_{C^{2}} .
$$

Proof.

Let us denote errors in process (5) by

$$
\bar{\psi}^{k}=\bar{u}^{k}-\bar{u}, k \in \mathrm{N} \cup\{0\} .
$$

In the iterative process, we obtain equalities

$$
\begin{gathered}
\left\langle C\left(\bar{\psi}^{1}-\bar{\psi}^{0}\right), C\left(\bar{\psi}^{1}-\bar{\psi}^{0}\right)\right\rangle=\left\langle-\Lambda_{11} \bar{\psi}_{1}^{0},-\Lambda_{11} \bar{\psi}_{1}^{0}\right\rangle \\
\left\langle C \bar{\psi}^{1}, C \bar{\psi}^{1}\right\rangle-2\left\langle C \bar{\psi}^{1}, C \bar{\psi}^{0}\right\rangle+\left\langle C \bar{\psi}^{0}, C \bar{\psi}^{0}\right\rangle=\left\langle\Lambda_{11} \bar{\psi}_{1}^{0}, \Lambda_{11} \bar{\psi}_{1}^{0}\right\rangle .
\end{gathered}
$$

Note that the inequality

$$
\left\langle C \bar{\psi}^{0}, C \bar{\psi}^{0}\right\rangle \geq\left\langle\Lambda_{11} \bar{\psi}_{1}^{0}, \Lambda_{11} \bar{\psi}_{1}^{0}\right\rangle
$$

holds. Also, note the fulfillment of the inequalities

$$
\begin{gathered}
\left\langle C \bar{\psi},{ }^{1} C \bar{\psi}^{1}\right\rangle-2\left\langle C \bar{\psi}^{1}, C \bar{\psi}^{0}\right\rangle \leq 0, \\
\left\langle C \bar{\psi}^{1}, C \bar{\psi}^{1}\right\rangle^{2} \leq 4\left\langle C \bar{\psi}^{1}, C \bar{\psi}^{0}\right\rangle^{2} \leq 4\left\langle C \bar{\psi}^{1}, C \bar{\psi}^{1}\right\rangle\left\langle C \bar{\psi}^{0}, C \bar{\psi}^{0}\right\rangle .
\end{gathered}
$$

After cancellation, we obtain inequalities

$$
\left\langle C \bar{\psi}^{1}, C \bar{\psi}^{1}\right\rangle \leq 4\left\langle C \bar{\psi}^{0}, C \bar{\psi}^{0}\right\rangle,\left\|\bar{\psi}^{1}\right\|_{C^{2}} \leq 2\left\|\bar{\psi}^{0}\right\|_{C^{2}},\left\|\bar{u}^{1}-\bar{u}\right\|_{C^{2}} \leq 2\left\|\bar{u}^{0}-\bar{u}\right\|_{C^{2}} .
$$

Theorem 2. For iterative extension method (5), convergence estimates

$$
\left\|\bar{u}^{k}-\bar{u}\right\|_{C^{2}} \leq \varepsilon\left\|\bar{u}^{0}-\bar{u}\right\|_{C^{2}}, \varepsilon=2\left(\beta_{2} / \beta_{1}\right)(\alpha / \gamma)^{k-1}, k \in \mathrm{N}
$$

hold. In a stronger norm than the energy norm, a sequence of relative errors is estimated from above by a converging geometric progression. 


\section{A. L. Ushakov}

Proof.

From the process, we get equalities for errors and residuals.

$$
\bar{\psi}^{k}=\bar{\psi}^{k-1}-\tau_{k} C^{-1} \Lambda_{\mathrm{II}} \bar{\psi}^{k-1}, \bar{r}^{k}=\bar{r}^{k-1}-\tau_{k} \Lambda_{\mathrm{II}} C^{-1} \bar{r}^{k-1}, \quad k \in \mathrm{N} \backslash 1 .
$$

Further, we minimize the residuals.

$0 \leq\left\langle\bar{r}^{k}, \bar{r}^{k}\right\rangle=\tau_{k}^{2}\left\langle\Lambda_{\mathrm{II}} C^{-1} \bar{r}^{k-1}, \Lambda_{\mathrm{II}} C^{-1} \bar{r}^{k-1}\right\rangle-2 \tau_{k}\left\langle\Lambda_{\mathrm{II}} C^{-1} \bar{r}^{k-1}, \bar{r}^{k-1}\right\rangle+\left\langle\bar{r}^{k-1}, \bar{r}^{k-1}\right\rangle$.

Choose parameters.

$$
\tau_{k-1}=\frac{\left\langle\Lambda_{\mathrm{II}} C^{-1} \bar{r}^{k-1}, \bar{r}^{k-1}\right\rangle}{\left\langle\Lambda_{\mathrm{II}} C^{-1} \bar{r}^{k-1}, \Lambda_{\mathrm{II}} C^{-1} \bar{r}^{k-1}\right\rangle}=\frac{\left\langle\bar{r}^{k-1}, \bar{\eta}^{k-1}\right\rangle}{\left\langle\bar{\eta}^{k-1}, \bar{\eta}^{k-1}\right\rangle}
$$

Note the presence of equality

$$
\tau_{k-1}=\frac{\left\langle\Lambda_{\mathrm{II}} C^{-1} \bar{r}^{k-1}, \bar{r}^{k-1}\right\rangle}{\left\langle\Lambda_{\mathrm{II}} C^{-1} \bar{r}^{k-1}, \Lambda_{\mathrm{II}} C^{-1} \bar{r}^{k-1}\right\rangle}=\frac{\left\langle\Lambda_{\mathrm{II}} \bar{w}^{k-1}, C \bar{w}^{k-1}\right\rangle}{\left\langle\Lambda_{\mathrm{II}} \bar{w}^{k-1}, \Lambda_{\mathrm{II}} \bar{w}^{k-1}\right\rangle} .
$$

Denote

$$
\Lambda_{\mathrm{I}} \bar{w}^{k-1}=\bar{a}, \Lambda_{\mathrm{II}} \bar{w}^{k-1}=\bar{b} .
$$

Note that the chosen parameters are positive.

$$
\tau_{k}=\frac{\langle\bar{b}, \bar{a}+\gamma \bar{b}\rangle}{\langle\bar{b}, \bar{b}\rangle}=\gamma-\frac{\langle\bar{a}, \bar{b}\rangle}{\langle\bar{b}, \bar{b}\rangle} \geq \gamma-\frac{\langle\bar{a}, \bar{a}\rangle^{1 / 2}\langle\bar{b}, \bar{b}\rangle^{1 / 2}}{\langle\bar{b}, \bar{b}\rangle} \geq \gamma-\frac{\langle\bar{a}, \bar{a}\rangle^{1 / 2}}{\langle\bar{b}, \bar{b}\rangle^{1 / 2}} \geq \gamma-\alpha>0 .
$$

Let us write the dot products with residuals under the chosen parameters.

$$
\left\langle\bar{r}^{k}, \bar{r}^{k}\right\rangle=\left\langle\bar{r}^{k-1}, \bar{r}^{k-1}\right\rangle-\frac{\left\langle\Lambda_{\mathrm{II}} C^{-1} \bar{r}^{k-1}, \bar{r}^{k-1}\right\rangle^{2}}{\left\langle\Lambda_{\mathrm{II}} C^{-1} \bar{r}^{k-1}, \Lambda_{\mathrm{II}} C^{-1} \bar{r}^{k-1}\right\rangle} .
$$

Also, write the ratio of the scalar products of the residuals.

$$
\begin{gathered}
q_{k}^{2}=\frac{\left\langle\bar{r}^{k}, \bar{r}^{k}\right\rangle}{\left\langle\bar{r}^{k-1}, \bar{r}^{k-1}\right\rangle}=1-\frac{\left\langle\Lambda_{\mathrm{II}} C^{-1} \bar{r}^{k-1}, \bar{r}^{k-1}\right\rangle^{2}}{\left\langle\Lambda_{\mathrm{II}} C^{-1} \bar{r}^{k-1}, \Lambda_{\mathrm{II}} C^{-1} \bar{r}^{k-1}\right\rangle\left\langle\bar{r}^{k-1}, \bar{r}^{k-1}\right\rangle}= \\
=\frac{\left\langle\Lambda_{\mathrm{II}} \bar{w}^{k-1}, \Lambda_{\mathrm{II}} \bar{w}^{k-1}\right\rangle\left\langle C \bar{w}^{k-1}, C \bar{w}^{k-1}\right\rangle-\left\langle\Lambda_{\mathrm{II}} \bar{w}^{k-1}, C \bar{w}^{k-1}\right\rangle^{2}}{\left\langle\Lambda_{\mathrm{II}} \bar{w}^{k-1}, \Lambda_{\mathrm{II}} \bar{w}^{k-1}\right\rangle\left\langle C \bar{w}^{k-1}, C \bar{w}^{k-1}\right\rangle}= \\
=\frac{\langle\bar{b}, \bar{b}\rangle\langle\bar{a}+\gamma \bar{b}, \bar{a}+\gamma \bar{b}\rangle-\langle\bar{b}, \bar{a}+\gamma \bar{b}\rangle^{2}}{\langle\bar{b}, \bar{b}\rangle\langle\bar{a}+\gamma \bar{b}, \bar{a}+\gamma \bar{b}\rangle} .
\end{gathered}
$$

Denote

$$
\langle\bar{a}, \bar{a}\rangle=a,\langle\bar{b}, \bar{b}\rangle=b,\langle\bar{a}, \bar{b}\rangle=z,
$$

then

$$
q_{k}^{2}=\frac{a b-z^{2}}{b\left(a+\gamma^{2} b+2 \gamma z\right)} \leq \max _{|z| \leq \sqrt{a b}} q_{k}^{2}(z)=q_{k}^{2}\left(\frac{-a}{\gamma}\right)=\frac{a}{\gamma^{2} b} \leq \frac{\alpha^{2}}{\gamma^{2}}=q^{2}
$$


taking into account that

$$
q_{k}^{2} \geq 0,\left(q_{k}^{2}(z)\right)_{z}^{\prime}=\frac{-2 \gamma(z+a / \gamma)(z+\gamma b)}{b\left(a+\gamma^{2} b+2 \gamma z\right)^{2}},-\gamma b<\frac{a+\gamma^{2} b}{2 \gamma}<-\sqrt{a b}<-\frac{a}{\gamma}<\sqrt{a b} .
$$

We establish inequalities

$$
\left\langle\Lambda_{\mathrm{II}} \bar{\psi}^{k}, \Lambda_{\mathrm{II}} \bar{\psi}^{k}\right\rangle \leq q^{2}\left\langle\Lambda_{\mathrm{II}} \bar{\psi}^{k-1}, \Lambda_{\mathrm{II}} \bar{\psi}^{k-1}\right\rangle,\left\langle\Lambda_{\mathrm{II}} \bar{\psi}^{k}, \Lambda_{\mathrm{II}} \bar{\psi}^{k}\right\rangle \leq q^{2(k-1)}\left\langle\Lambda_{\mathrm{II}} \bar{\psi}^{1}, \Lambda_{\mathrm{II}} \bar{\psi}^{1}\right\rangle, k \in \mathrm{N} \backslash\{1\} .
$$

Taking into account that $\left\langle C \bar{\psi}^{k}, C \bar{\psi}^{k}\right\rangle \leq \beta_{1}^{-2}\left\langle\Lambda_{\mathrm{II}} \bar{\psi}^{k}, \Lambda_{\mathrm{II}} \bar{\psi}^{k}\right\rangle,\left\langle\Lambda_{\mathrm{II}} \bar{\psi}^{1}, \Lambda_{\mathrm{II}} \bar{\psi}^{1}\right\rangle \leq \beta_{2}^{2}\left\langle C \bar{\psi}^{1}, C \bar{\psi}^{1}\right\rangle \leq 4 \beta_{2}^{2}\left\langle C \bar{\psi}^{0}, C \bar{\psi}^{0}\right\rangle$, we obtain the inequality that gives an estimate for the convergence of the method of iterative extensions:

$$
\left\langle C \bar{\psi}^{k}, C \bar{\psi}^{k}\right\rangle \leq 4 \beta_{1}^{-2} \beta_{2}^{2} q^{2(k-1)}\left\langle C \bar{\psi}^{0}, C \bar{\psi}^{0}\right\rangle
$$

\section{Algorithmic Implementation, Programmable Algorithm for the Method of Iterative Extensions}

When choosing iterative parameters, we apply the method of minimum residuals.

I. Choose an initial approximation and an iterative parameter

$$
\forall \bar{u}^{0} \in \bar{V}_{1}, \tau_{0}=1 .
$$

II. Find the residual

$$
\bar{r}^{k-1}=B \bar{u}^{k-1}-\bar{f}, k \in \mathrm{N} .
$$

III. Calculate the norm of absolute error squared

$$
\mathrm{E}_{k-1}=\left\langle\bar{r}^{k-1}, \bar{r}^{k-1}\right\rangle, k \in \mathrm{N} .
$$

IV. Find the correction

$$
\bar{w}^{k-1}: C \bar{w}^{k-1}=\bar{r}^{k-1}, k \in \mathrm{N} .
$$

V. Find the equivalent residual

$$
\eta^{k-1}=B \bar{w}^{k-1}, k \in \mathrm{N} \backslash\{1\} .
$$

VI. Find the iteration parameter

$$
\tau_{k-1}=\left\langle\bar{r}^{k-1}, \bar{\eta}^{k-1}\right\rangle /\left\langle\bar{\eta}^{k-1}, \bar{\eta}^{k-1}\right\rangle=\left\langle\bar{r}_{2}^{k-1}, \bar{\eta}_{2}^{k-1}\right\rangle /\left\langle\bar{\eta}_{2}^{k-1}, \bar{\eta}_{2}^{k-1}\right\rangle, k \in \mathrm{N} \backslash\{1\} .
$$

VII. Find the next approximation

$$
\bar{u}^{k}=\bar{u}^{k-1}-\tau_{k-1} \bar{w}^{k-1}, k \in \mathrm{N} .
$$

VIII. Check the fulfillment of the criterion for stopping iterations by a given estimate of the relative error

$$
\mathrm{E}_{k-1} \leq \mathrm{E}_{0} \mathrm{E}, k \in \mathrm{N} \backslash\{1\}, \mathrm{E} \in(0 ; 1) .
$$




\section{An Example Using the Iterative Extension Method}

Consider the problem in the following domains:

$$
\Pi=(0 ; 8) \times(0 ; b), \Omega_{1}=(0 ; 8) \times(0 ; 4), \Omega_{\mathrm{II}}=(0 ; 8) \times(4 ; b) .
$$

We assume that the domains have boundaries

$$
\begin{gathered}
\Gamma_{0}=\emptyset, \Gamma_{1}=(0 ; 8) \times\{b\}, \Gamma_{2}=(0 ; 8) \times\{0\} \cup\{0,8\} \times(0 ; b), \Gamma_{3}=\emptyset, \\
\Gamma_{1,0}=(0 ; 8) \times\{4\}, \Gamma_{1,1}=\emptyset, \Gamma_{1,2}=(0 ; 8) \times\{0\} \cup\{0,8\} \times(0 ; 4), \Gamma_{1,3}=\emptyset, \\
\Gamma_{\mathrm{II}, 0}=\emptyset, \Gamma_{\mathrm{II}, 1}=(0 ; 8) \times\{b\}, \Gamma_{\mathrm{II}, 2}=\{0,8\} \times(4 ; b), \Gamma_{\mathrm{II}, 3}=(0 ; 8) \times\{4\} .
\end{gathered}
$$

Take the right side with the coefficient of the equation

$$
\breve{f}_{1}(x ; y)=6,(x ; y) \in(0 ; 8) \times(0 ; 4), a_{\mathrm{II}}(x ; y)=1,(x ; y) \in(0 ; 8) \times(4 ; b) .
$$

Let us give a solution to the problem.

$$
\breve{u}_{1}(x ; y)=(y+4)^{2}(y-4)^{2} / 4,(x ; y) \in(0 ; 8) \times(0 ; 4) .
$$

When sampling, choose $h_{1}=h_{2}=8 /(n+2), b=4(2 n+1) /(n+2), n=36,42, \ldots, 102$.

When calculating in the method of iterative extensions with a zero initial approximation, i.e. with a unit relative error, the process stops at the fourth iteration if an estimate for a relative error is considered to be one thousandth. Fig. 1 presents the graphs of the first approximation and the exact solution to the continued problem in the section by a plane perpendicular to the abscissa axis. The graphs of the second, third and fourth approximations practically coincide with the graph of the exact solution.

$$
n=102, \mathrm{E}=0,001, u_{i, j}^{1} \geq u_{i, j}^{2} \approx u_{i, j}^{3} \approx u_{i, j}^{4} \approx u_{i, j} \geq u_{i, j}^{0}=0, k=4 .
$$

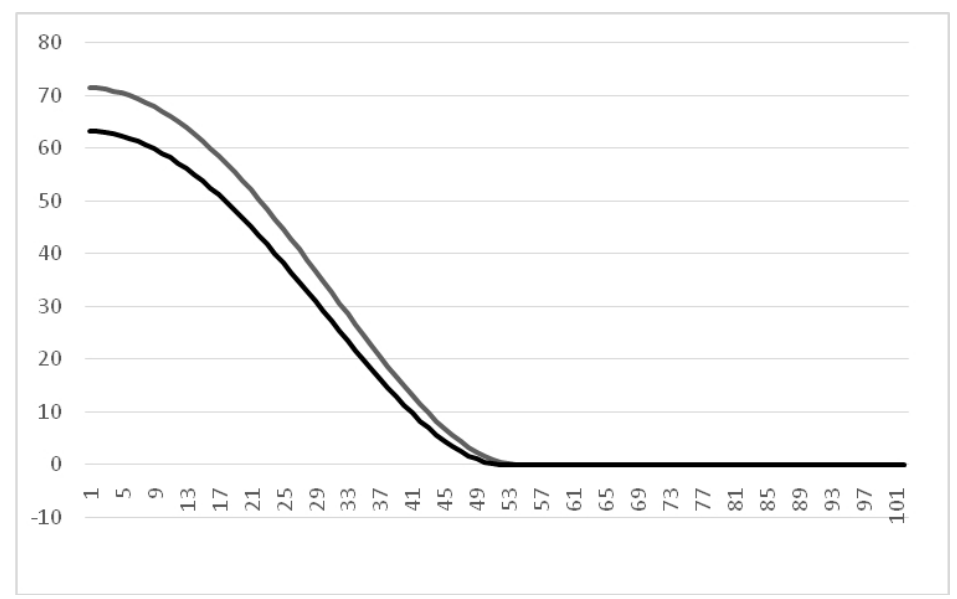

Fig. 1. First approximation and exact solution

Note that at the last fourth iteration, on the smallest of the grids used, there is fulfillment of inequalities that characterize the accuracy of the numerical solution to the original problem in the case under consideration.

$n=102, \mathrm{E}=0,001, \max _{\left(x_{i} ; y_{j}\right) \in \Omega_{1}} \frac{\left|u_{i, j}^{k}-u_{i, j}\right|}{\left|u_{i, j}\right|} \leq 0,04, \quad \frac{\max _{\left(x_{i} ; y_{j}\right) \in \Omega_{1}}\left|u_{i, j}^{k}-u_{i, j}\right|}{\max _{\left(x_{i} ; y_{j}\right) \in \Omega_{1}}\left|u_{i, j}\right|} \leq 0,0004, k=4$. 


\section{References}

1. Aubin J. P. Approximation of Elliptic Boundary-value Problems. New York, WileyInterscience, 1972.

2. Sorokin S. B. An Economical Algorithm for Numerical Solution of the Problem of Identifying the Right-Hand Side of the Poisson Equation. Journal of Applied and Industrial Mathematics, 2018, vol. 12, issue 2, pp. 362-368. DOI: $10.1134 /$ S1990478918020163

3. Sorokin S. B. An Efficient Direct Method for the Numerical Solution to the Cauchy Problem for the Laplace Equation. Numerical Analysis and Applications, 2019, vol. 12, issue 1, pp. 87-103. DOI: 10.1134/S1995423919010075

4. Ushakov A. L. Investigation of a Mixed Boundary Value Problem for the Poisson Equation. 2020 International Russian Automation Conference (RusAutoCon), Sochi, Russia, 2020, pp. 273-278. DOI: 10.1109/RusAutoCon49822.2020.9208198

5. Ushakov A. L. About Modelling of Deformations of Plates. Bulletin of the South Ural State University. Ser. Mathematical Modelling, Programming \& Computer Software, 2015, vol. 8, no. 2, pp. 138-142. (in Russian). DOI: 10.14529/mmp150213

6. Matsokin A. M., Nepomnyaschikh S. V. The Fictitious-Domain Method and Explicit Continuation Operators. Computational Mathematics and Mathematical Physics, 1993, vol. 33, no. 1, pp. 45-59.

7. Marchuk G. I., Kuznetsov Yu. A., Matsokin A. M. Fictitious Domain and Domain Decomposition Methods. Russian Journal of Numerical Analysis and Mathematical Modelling, 1986, vol. 1, issue 1, pp. 3-35. DOI: 10.1515/rnam.1986.1.1.3

8. Bank R. E., Rose D. J. Marching Algorithms for Elliptic Boundary Value Problems. I: The Constant Coefficient Case. SIAM Journal on Numerical Analysis, 1977, vol. 14, issue 5, pp. 792-829. DOI: 10.1137/0714055

9. Manteuffel T. An Incomplete Factorization Technique for Positive Definite Linear Systems. Mathematics of Computation, 1980, vol. 34, pp. 473-479. DOI: $10.1090 /$ S0025-5718-1980-0559197-0

10. Swarztrauber P. N. A Direct Method for Discrete Solution of Separable Elliptic Equations. SIAM Journal on Numerical Analysis, 1974, vol. 11, issue 6, pp. 1136-1150. DOI: $10.1137 / 0711086$

11. Swarztrauber P. N. The Methods of Cyclic Reduction, Fourier Analysis and the FACR Algorithm for the Discrete Solution of Poisson's Equation on a Rectangle. SIAM Review, 1977, vol. 19, no. 3, pp. 490-501. DOI: 10.1137/1019071

Andrey L. Ushakov, PhD (Math), Docent, Senior Research Officer at the Department of Mathematical and Computer Modelling, South Ural State University (Chelyabinsk, Russian Federation), ushakoval@susu.ru.

Received February 17, 2021. 


\title{
ЧИСЛЕННЫЙ АНАЛИЗ СМЕШАННОЙ КРАЕВОЙ ЗАДАЧИ ДЛЯ УРАВНЕНИЯ СОФИ ЖЕРМЕН
}

\author{
А. Л. Уиаков
}

\begin{abstract}
Уравнение Софи Жермен в вариационном виде рассматривается на плоской ограниченной области при смешанных краевых условиях. Производится продолжение исходной задачи на прямоугольную область по границе с краевыми условиями Дирихле. Производится дискретизация продолженной задачи с помощью метода конечных элементов. Приводится метод фиктивных компонент, который для абсолютной ошибки имеет скорость сходимости как у геометрической прогрессии. Для численного решения продолженной задачи используется метод итерационных расширений получающийся как обобщение метода фиктивных компонент. Метод итерационных расширений для относительной ошибки имеет скорость сходимости как у геометрической прогрессии в более сильной норме, чем энергетическая норма задачи. Выбор итерационных параметров проводится на основе метода минимальных невязок. Приводится алгоритмическая реализация метода итерационных расширений с автоматическим выбором итерационных параметров. В алгоритме приводится критерий и для автоматической остановки итерационного процесса при достижении заданной оценки относительной ошибки. При программной реализация этого алгоритма не требуется дополнительных усложнений связанных со сложностью геометрии области, порядком дифференциального уравнения и наличием краевых условий Дирихле. Рассматривается пример расчетов по методу итерационных расширений при численном решении частной задачи. При счете используется условие выполнения оценки для относительной ошибки в более сильной норме, чем норма порождаемая оператором задачи. Но рассматриваются относительные ошибки для полученного численного решения примера у исходной задачи еще и другими способами. Например, проводится поточечное вычисление относительной ошибки по узлам сетки. Получается, что нескольких итераций достаточно, что бы иметь относительную ошибку только в несколько процентов даже при различных способах ее вычисления. Вычислительные эксперименты на практике подтверждают оптимальность метода, указанную в теории.
\end{abstract}

Ключевые слова: уравнение Соби Жермен; метод фиктивных компонент; метод итерационных расширений.

\section{Литература}

1. Aubin, J. P. Approximation of Elliptic Boundary-Value Problems / J. P. Aubin. New York: Wiley-Interscience, 1972.

2. Сорокин, С. Б. Экономичный алгоритм для численного решения задачи идентификации правой части уравнения Пуассона / С. Б. Сорокин / / Сибирский журнал индустриальной математики. - 2018. - Т. 21, вып. 2. - С. 101-107.

3. Сорокин, С. Б. Экономичный прямой метод численного решения задачи Коши для уравнения Лапласа / С. Б. Сорокин // Сибирский журнал вычислительной математики. - 2019. - Т. 22, вып. 1. - С. 99-117.

4. Ushakov, A. L. Investigation of a Mixed Boundary Value Problem for the Poisson Equation / A. L. Ushakov // 2020 International Russian Automation Conference (RusAutoCon). - Sochi, Russia: IEEE, 2020. - P. 273-278. 
5. Ушаков, А. Л. О моделировании деформаций пластин / А. Л. Ушаков // Вестник ЮУрГУ. Серия: Математическое моделирование и программирование. - 2015. T. 8, № 2. - C. 138-142.

6. Мацокин, А. М. Метод фиктивного пространства и явные операторы продолжения / А. М. Мацокин, С. В. Непомнящих // Журнал вычислительной математики и математической физики. - 1993. - Т. 33, № 1. - С. 52-68.

7. Marchuk, G. I. Fictitious Domain and Domain Decomposition Methods G. I. Marchuk, Yu. A. Kuznetsov, A. M. Matsokin // Russian Journal of Numerical Analysis and Mathematical Modelling. - 1986. - V. 1, iss. 1. - P. 3-35.

8. Bank, R. E. Marching Algorithms for Elliptic Boundary Value Problems. I: The Constant Coefficient Case / R. E. Bank, D. J. Rose // SIAM Journal on Numerical Analysis. - 1977. - V. 14, iss. 5. - P. 792-829.

9. Manteuffel, T. An Incomplete Factorization Technique for Positive Definite Linear Systems / T. Manteuffel // Mathematics of Computation. - 1980. - V. 34. P. 473-479.

10. Swarztrauber, P. N. A Direct Method for Discrete Solution of Separable Elliptic Equations / P. N. Swarztrauber // SIAM Journal on Numerical Analysis. - 1974. - V. 11, iss. 6. - P. 1136-1150.

11. Swarztrauber, P. N. The Methods of Cyclic Reduction, Fourier Analysis and the FACR Algorithm for the Discrete Solution of Poisson's Equation on a Rectangle / P. N. Swarztrauber // SIAM Review. - 1977. - Vol. 19, № 3. - P. 490-501.

Уиаков Андрей Леонидович, кандидат физико-математических наук, дочент, старший научный сотрудник кафедры математического и компьютерного моделирования, Южно-Уральский государственный университет (г. Челябинск, Российская Федераuия), ushakoval@susu.ru.

Поступила в редакцию 17 февраля 2021 г. 